\title{
Efeito da Seleção para Peso Pós-desmama sobre Indicadores da Eficiência Produtiva de Vacas da Raça Nelore ${ }^{1}$
}

\author{
Josineudson Augusto II de Vasconcelos Silva², Alexander George Razook ${ }^{3,5}$, Humberto \\ Tonhati $^{4,5}$, Leopoldo Andrade de Figueiredo ${ }^{3}$
}

\begin{abstract}
RESUMO - O objetivo deste estudo foi avaliar indicadores da eficiência produtiva de vacas Nelore selecionadas para peso pósdesmama, enfocados nas relações peso bezerro e peso da vaca, nos rebanhos controle ( $\mathrm{NeC}$ ), não-selecionados para peso pós-desmama, seleção (NeS) e tradicional (NeT). As análises envolveram 3929 e 3906 pesos dos bezerros aos 120 (P120) e 210 (P210) dias de idade, respectivamente. Os pesos das vacas àquelas idades também foram considerados, obtendo-se 3824 (PV120) e 3777 (PV210) registros, respectivamente, para 120 e 210 dias. A análise incluiu 183, 375 e 554 vacas dos rebanhos NeC, NeS e NeT, respectivamente. Para as análises utilizou-se o procedimento GLM/SAS, em modelos que incluíram os efeitos fixos de rebanho, ano e mês de nascimento, sexo do bezerro, idade da vaca ao parto e as interações rebanho x ano de nascimento e ano x mês de nascimento. Todos os efeitos foram significativos. Os resultados mostraram respostas correlacionadas positivas na seleção para peso pós-desmama nos pesos dos bezerros e, em menor magnitude, no peso das vacas. As relações peso bezerro e peso da vaca apresentaram valores médios ajustados de $249,0 \pm 2,1 ; 253,7 \pm 1,6$; e $255,8 \pm 1,2 \mathrm{~g} / \mathrm{kg}$ para R120 e $362,9 \pm 2,8 ; 368,5 \pm 2,1$; e $374,6 \pm 1,7$ para NeC, NeS e NeT em R210, respectivamente, indicando que, nas duas idades, os rebanhos $\mathrm{NeS}$ e $\mathrm{NeT}$ produziram mais quilogramas de bezerro por quilogramas de vaca, quando comparados ao $\mathrm{NeC}$.
\end{abstract}

Palavras-chave: habilidade materna, Nelore, peso pós-desmama, seleção, vacas

\section{Effects of Selection for Post Weaning Weight on Nellore Cow Productivity Efficiency Indicators}

\begin{abstract}
The objective of this study was to evaluate the productivity efficiency indicators of cows selected for post-weaning weight, with emphasis in the calf-cow weight ratios, in a controlled herd $(\mathrm{NeC})$, unselected for post-weaning weight, selection (NeS) and conventional (NeT). The analysis involved 3929 and 3906 calves weights at the 120 (P120) and 210 (P210) days of age, respectively. The cow weights at those ages were also considered, and the recorded numbers were 3824 (PV120) and 3777 (PV210), respectively, for 120 and 210 days. The analysis included 183, 375 and 554 cows from $\mathrm{NeC}, \mathrm{NeS}$ and NeT, respectively. The SAS/ GLM procedure was used in the data analyses. The model included the effects of herd, year and month of birth, and sex of calf, age of cow at calving, and the interactions herd x year, and year x month of birth. All effects were significant. The results showed a positive correlated response in the selection for post weaning on the calf weights and, low response, on cow weights. The calf weight/cow weight ratios showed average adjusted values of $249.0 \pm 2.1,253.7 \pm 1.6$ and $255.8 \pm 1.2 \mathrm{~g} / \mathrm{kg}$ for R120, and 362.9 $\pm 2.8,368.5 \pm 2.1$ and $374.6 \pm 1.7 \mathrm{~g} / \mathrm{kg}$ for $\mathrm{NeC}, \mathrm{NeS}$ and $\mathrm{NeT}$ at R210, respectively, and indicated that, at both ages, the NeS and NeT herds produced more $\mathrm{kg}$ of calf by $\mathrm{kg}$ of cow when compared to $\mathrm{NeC}$.
\end{abstract}

Key Words: cows, maternal hability, Nellore, post-weaning weight, selection

\section{Introdução}

A avaliação da eficiência produtiva é de suma importância para a exploração de bovinos de corte e pode ser quantificada pelo número de bezerros desmamados ou pela quantidade de quilogramas de bezerros desmamados por vaca/ano, os quais refletem sobre a rentabilidade do rebanho (URICK et al., 1971; MORRIS e WILTON, 1976; DINKEL et al., 1978; DAVIS et al., 1983; RAHNEFELD et al., 1993; e ALENCAR et al. 1996).

O objetivo do presente trabalho foi avaliar a eficiência produtiva de vacas da raça Nelore, comparando três rebanhos selecionados diferente-

\footnotetext{
${ }^{1}$ Parte da Dissertação de Mestrado em Zootecnia - FCAVJ/UNESP - Jaboticabal, SP. Apoio: CNPq.

2 Aluno de Doutorado em Zootecnia - Dept. Melh. e Nutr. Anim. - FMVZ/UNESP - Botucatu, SP.

3 Pesquisador da Estação Experimental de Zootecnia de Sertãozinho, Instituto de Zootecnia, Sertãozinho, SP. E.mail: sertaozinho@.izsp.br

${ }^{4}$ Professor do Departamento de Zootecnia - FCAVJ/UNESP - Jaboticabal, SP.

${ }^{5}$ Bolsista CNPq.
} 
mente com base no peso pós-desmama. A eficiência foi medida pela relação entre o peso do bezerro e o peso da vaca, em duas idades dos bezerros, aos quatro e aos sete meses (desmama).

\section{Material e Métodos}

O presente estudo foi desenvolvido com informações referentes aos rebanhos da raça Nelore da Estação Experimental de Zootecnia de Sertãozinho (EEZS), do Instituto de Zootecnia da Secretária da Agricultura e Abastecimento do Estado de São Paulo, situada na região norte do Estado de São Paulo, distando aproximadamente $10 \mathrm{~km}$ da cidade de Sertãozinho e $30 \mathrm{~km}$ de Ribeirão Preto. A estação está localizada a $21^{\circ} 08^{\prime}$ de latitude sul e $47^{\circ} 59^{\prime}$ de longitude a oeste de Greenwich, com altitude média de $548 \mathrm{~m}$. O clima é tropical úmido e corresponde ao tipo Aw, segundo a classificação de Köeppen, com estação chuvosa no verão e seca no inverno.

Os procedimentos de implantação do projeto de seleção da EEZS foram detalhados por RAZOOK et al. (1993). Em linhas gerais, em 1980, o rebanho da raça Nelore foi dividido em dois rebanhos, controle $(\mathrm{NeC})$ e seleção (NeS). O rebanho NeS é constituído de 120 fêmeas e seis touros e o $\mathrm{NeC}$, de 60 fêmeas e quatro touros. O rebanho tradicional (NeT) foi formado com vacas remanescentes da formação dos rebanhos controle e seleção e, hoje, é constituído por 150 matrizes e oito touros.

Os registros obtidos para cada vaca incluíram a identificação, o peso ao término da estação de monta, o qual representa o peso da vaca à idade de 120 dias do produto da estação anterior, o peso da vaca ao desmamar e de seu bezerro, bem como a sua idade ao parto. Os registros obtidos para os bezerros foram a identificação, a data de nascimento, o sexo e os pesos e datas tomados próximos dos 120 e 210 dias de idade (desmama). As progênies que forneceram os dados utilizados neste estudo nasceram de 1981 a 1996 principalmente nos meses de setembro, outubro e novembro.

Os rebanhos $\mathrm{NeC}, \mathrm{NeS}$ e $\mathrm{NeT}$ formaram os seguintes números de observações: $\mathrm{N}=3929$ para o peso aos 120 dias de idade do bezerro (P120); $\mathrm{N}=3906$ para o peso aos 210 dias de idade (P210); $\mathrm{N}=3824$ para o peso da vaca aos 120 dias de idade do bezerro (PV120); e $\mathrm{N}=3777$ para o peso da vaca aos 210 dias de idade do bezerro (PV210). No total foram 1112 vacas distribuídas, respectivamente, em 183, 375 e 554 para os rebanhos NeC, NeS e NeT.
Para a padronização dos pesos dos bezerros e das vacas, utilizou-se a fórmula com base na taxa de crescimento individual (RAZOOK et al., 1993). As relações entre o peso do bezerro e o peso da vaca foram obtidas aos 120 (R120) e 210 (R210) dias de idade dos bezerros, por meio da seguinte expressão: $\mathrm{R}=\mathrm{PP} * 1000 / \mathrm{PPV}$, expresso em gramas de bezerro por quilo de vaca, em que $\mathrm{R}=$ relação do peso do bezerro sobre o peso da vaca, aos 120 e 210 dias de idade do bezerro; $\mathrm{PP}=$ peso do bezerro padronizado para 120 e 210 dias de idade; e PPV = peso da vaca padronizado às mesmas idades.

Para as análises estatísticas, utilizou-se o procedimento GLM, contido no pacote estatístico SAS (1992). O modelo estatístico incluiu os efeitos fixos de rebanho, ano, mês e sexo do bezerro, a idade da vaca ao parto e as interações entre rebanho e o ano de nascimento e entre o ano e o mês de nascimento do animal. Esse modelo foi utilizado para os pesos dos bezerros e das vacas, bem como para as relações no intuito de se investigar o grau de influência dos principais fatores de variação sobre as características.

Foram também obtidas tendências lineares das características estudadas, pelo método da regressão das características sobre o ano de nascimento do bezerro, dentro de cada rebanho.

\section{Resultados e Discussão}

O número de observações, as médias observadas, os erros-padrão e os coeficientes de variação para os pesos dos bezerros (P120 e P210) e das vacas (PV120 e PV210) e para as relações (R120 e R210), nos rebanhos $\mathrm{NeC}, \mathrm{NeS}$ e $\mathrm{NeT}$, são apresentados na Tabela 1.

O P120 apresentou médias observadas variando entre 113,6 $\pm 0,6 \mathrm{~kg}(\mathrm{NeC})$ e 120,8 $\pm 0,6 \mathrm{~kg}(\mathrm{NeS})$, estando próximo às apresentadas por LÔBO et al. (1997), no sumário de avaliação genética de animais da raça Nelore, com média de $124,3 \mathrm{~kg}$ para machos e $114,8 \mathrm{~kg}$ para fêmeas.

Para o P210, as médias variaram de $167,5 \pm 0,9 \mathrm{~kg}$ $(\mathrm{NeC})$ a $180,3 \pm 0,7 \mathrm{~kg}(\mathrm{NeT})$ e foram superiores às obtidas na literatura para animais da raça Nelore, criados a pasto (PEROTTO et al., 1996; MAGNABOSCO et al., 1996; EUCLIDES FILHO et al., 1996; MARTINS FILHO et al., 1997; SOUZA et al., 1997; e FERRAZ FILHO et al., 1997), e próximas às obtidas por MACHADO et al. (1997) e ALENCAR et al. (1997).

Os PV120 e PV210 apresentaram médias com 
1022 Rev. bras. zootec.

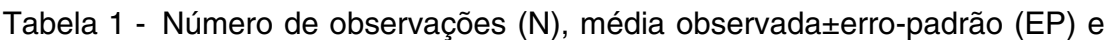
coeficiente de variação (CV) para características P120, P210, PV120, PV210, R120, e R210, nos rebanhos NeC, NeS e NeT

Table 1 - Number of observations (N), mean \pm standard error (SE) and coefficient of variation (CV) for the traits P120, P210, PV120, PV210, R120 e R210 for herds $\mathrm{NeC}, \mathrm{NES}, \mathrm{NeT}$

\begin{tabular}{lcrcc}
\hline Características $^{1}$ & $\begin{array}{c}\text { Rebanho } \\
\text { Traits }^{1}\end{array}$ & $\mathrm{~N}$ & $\begin{array}{c}\text { Média } \pm \mathrm{EP} \\
\text { Herd }\end{array}$ & CV(\%) \\
\hline \multirow{3}{*}{ P120 } & $\mathrm{NeC}$ & 644 & $113,6 \pm 0,6$ & 14,2 \\
& $\mathrm{NeS}$ & 1291 & $120,8 \pm 0,6$ & 17,2 \\
& $\mathrm{NeT}$ & 1994 & $119,3 \pm 0,4$ & 15,9 \\
P210 & $\mathrm{NeC}$ & 642 & $167,5 \pm 0,9$ & 15,0 \\
& $\mathrm{NeS}$ & 1284 & $179,0 \pm 0,9$ & 17,4 \\
& $\mathrm{NeT}$ & 1980 & $180,3 \pm 0,7$ & 16,1 \\
PV120 & $\mathrm{NeC}$ & 626 & $451,4 \pm 2,2$ & 12,3 \\
& $\mathrm{NeS}$ & 1251 & $469,8 \pm 1,6$ & 11,9 \\
& $\mathrm{NeT}$ & 1947 & $471,2 \pm 1,2$ & 11,1 \\
PV210 & $\mathrm{NeC}$ & 617 & $455,4 \pm 2,2$ & 11,9 \\
& $\mathrm{NeS}$ & 1237 & $479,2 \pm 1,6$ & 11,6 \\
& $\mathrm{NeT}$ & 1923 & $484,5 \pm 1,2$ & 10,7 \\
R120 & $\mathrm{NeC}$ & 626 & $255,8 \pm 1,8$ & 17,7 \\
& $\mathrm{NeS}$ & 1251 & $260,2 \pm 1,3$ & 18,2 \\
& $\mathrm{NeT}$ & 1947 & $256,2 \pm 1,2$ & 19,9 \\
R210 & $\mathrm{NeC}$ & 617 & $375,2 \pm 2,8$ & 18,4 \\
& $\mathrm{NeS}$ & 1237 & $380,1 \pm 2,0$ & 18,7 \\
& $\mathrm{NeT}$ & 1923 & $376,6 \pm 1,6$ & 18,2 \\
\hline
\end{tabular}

1 P120 e P210 (kg): pesos dos bezerros aos 120 e 210 dias de idade, respectivamente; PV120 e PV210 (kg): pesos das vacas nas respectivas idades dos bezerros; R120 e R210 $(\mathrm{g} / \mathrm{kg}): \mathrm{P} 120 * 1000 / \mathrm{PV} 120$ e P210*1000/PV210, respectivamente.

2 NeC: rebanho controle não-selecionado; $\mathrm{NeS}$ e NeT: rebanhos selecionados para peso pós-desmama.

1 P120 e P210 (kg): calves weights at 120 and 210 days of age; PV120 and PV210 (kg): cows weights at correspondent ages of calves; R120 and R210 (g/kg): P120*1000/PV120 and P210*1000/PV210 respectively.

$2 \mathrm{NeC}$ : Unselected control population; NeS e NeT: Selected Nellore lines for postweaning weight.

amplitude de variação de $451,4 \pm 2,2 \mathrm{~kg}(\mathrm{NeC})$ a $471,2 \pm 1,2 \mathrm{~kg}(\mathrm{NeT})$ e de $455,4 \pm 2,2 \mathrm{~kg}(\mathrm{NeC})$ a $484,5 \pm 1,2 \mathrm{~kg}(\mathrm{NeT})$, respectivamente. A única citação encontrada para a característica foi de ALVES FILHO e RESTLE (1996), que apresentaram média ajustada de $319,0 \mathrm{~kg}$ para o peso da vaca na desmama dos produtos.

Para a relação $\mathrm{R} 120$, as médias variaram entre $255,8 \pm 1,8(\mathrm{NeC})$ e $260,2 \pm 1,3 \mathrm{~g} / \mathrm{kg}(\mathrm{NeS})$, não tendo sido encontrados trabalhos na literatura referindo-se a esta característica.

As médias para R210 variaram entre $375,2 \pm 2,8 \mathrm{~g} / \mathrm{kg}$ $(\mathrm{NeC})$ e $380,1 \pm 2,0(\mathrm{NeS}) \mathrm{g} / \mathrm{kg}$, as quais foram ligeiramente inferiores às verificadas por OLIVEIRA et al. (1995) e ALENCAR et al. (1997), com médias estimadas de 437,0 e 427,0 $\pm 2 \mathrm{~g} / \mathrm{kg}$, respectivamente, ambos com animais da raça Nelore, porém com relações considerando o peso da vaca ao parto, o qual é normalmente inferior ao da desmama.

Constam da Tabela 2 as análises de variância para as relações $\mathrm{R} 120$ e $\mathrm{R} 210$. $\mathrm{O}$ ano de nascimento do bezerro foi importante fonte de variação $(\mathrm{P}<0,01)$, coincidindo com os resultados de URICK et al. (1971), em Angus, Charolês e Hereford, DINKEL et al. (1992) e OLIVEIRA et al. (1995), na raça Nelore, e ALENCAR et al. (1997), em vacas Nelore e cruzadas. A fonte de variação mês de nascimento também foi importante $(\mathrm{P}<0,01)$ para as relações $\mathrm{R} 120 \mathrm{e}$ R210. Flutuações climáticas de um mês para o outro, em temperatura, precipitação, umidade relativa e outros fatores, possivelmente influíram nas condições de criação nos diferentes meses e estão relacionadas com o estado físico das vacas e, conseqüentemente, seu peso e o do bezerro.

Resultados indicando influências significativas de mês ou época do nascimento sobre a relação peso do bezerro pelo peso da vaca, foram relatados por ALENCAR (1988) nas raças Canchim e Nelore. O mesmo foi relatado por OLIVEIRA et al. (1995), em animais da raça Nelore, e ALENCAR et al. (1997), 
em vacas Nelore e cruzadas.

O sexo do bezerro também apresentou efeito $(\mathrm{P}<0,01)$ sobre as características estudadas, sendo que as médias ajustadas dos machos foram maiores, com diferenças em relação às fêmeas de 20,3 e $38,1 \mathrm{~g} / \mathrm{kg}$ nas relações R120 e R210, respectivamente. Outros autores também observaram efeito significativo do sexo do bezerro, como FRAHM e MARSHALL (1985), em animais cruzados de várias raças, ALENCAR (1988), em Canchim e Nelore, DINKEL et al. (1992) e OLIVEIRA et al. (1995), em animais Nelore, e ALENCAR et al. (1997), em animais Nelore e cruzados.

As relações R120 e R210 foram influenciadas $(\mathrm{P}<0,01)$ pela idade da vaca ao parto, havendo tendências de diminuírem com o aumento da idade das vacas. Este resultado demonstra que pode haver a necessidade de fatores de correção dessas relações para essa fonte de variação, pois as vacas mais novas, apesar de apresentarem pesos ao desmame menores, mostram vantagens devido seu menor peso, quando comparadas com vacas mais velhas. Efeitos significativos da idade da vaca ao parto foram também encontrados por URICK et al. (1971), em animais das raças Angus, Charolês e Hereford, FRAHM e MARSHALL (1985,) em animais mestiços, DINKEL et al. (1992), RAHNEFELD et al. (1993) e ALENCAR et al. (1997), em vacas Nelore e cruzadas. Também as interações entre rebanho e ano de

Tabela 2 - Análises de variância para as relações R120 e R210 Tabela 2 - Analyses of variance for $R 120$ and $R 210$ ratios

\begin{tabular}{|c|c|c|c|}
\hline \multirow{3}{*}{$\begin{array}{l}\text { Fontes de variação }{ }^{1} \\
\text { Source of variation }\end{array}$} & \multirow{3}{*}{$\begin{array}{l}\mathrm{g} \mathrm{l}^{2} \\
d f^{2}\end{array}$} & \multirow{2}{*}{\multicolumn{2}{|c|}{$\begin{array}{l}\text { Quadrado médio } \\
\text { Mean square }\end{array}$}} \\
\hline & & & \\
\hline & & R120 & $\mathrm{R} 210$ \\
\hline REB & 2 & $9343^{* *}$ & $28.836^{* * *}$ \\
\hline ANB & 15 & $12.008^{* *}$ & $45.583^{* *}$ \\
\hline MNB & 2 & $14.961^{* *}$ & $36.435^{* *}$ \\
\hline SXB & 1 & $34.3377^{* *}$ & $1.327 .584^{* *}$ \\
\hline IVP & 10 & $38.526^{* *}$ & $10.4857^{* *}$ \\
\hline REB X ANB & 30 & $3938^{* *}$ & $12.121^{* *}$ \\
\hline REB X MNB & 30 & $4587^{* *}$ & $9026^{* *}$ \\
\hline Resíduo $^{3}$ & & 2101(3733) & $3761(3686)$ \\
\hline
\end{tabular}

Error

${ }^{1}$ REB, ANB, MNB, SXB, IVP, REB x ANB, REB x MNB são rebanho, ano de nascimento, mês de nascimento, sexo do animal, idade da vaca ao parto e as interações, respectivamente.

2 Graus de liberdade.

${ }^{3}$ Entre parênteses estão os graus de liberdade dos resíduos para cada característica.

1 REB, ANB, MNB, SXB, IVP, REBX ANB, REBXMNBare: herd, yearand month of birth, seX, age of cow at parturition and the interactions.

2 Degrees of freedom.

3 The degrees of freedom of errors for each trait are between parenthesis. nascimento e entre ano e mês de nascimento do animal foram significativas $(\mathrm{P}<0,01)$ para as relações R120 e R210. Em programas de seleção com diferentes linhagens (controle e seleção, por exemplo), a interação rebanho por ano de nascimento é quase sempre significativa, em virtude do próprio progresso genético alcançado nas diferentes características.

A fonte de variação rebanho influenciou $(\mathrm{P}<0,01)$ as duas relações estudadas, demonstrando que o processo de seleção aplicado no decorrer dos 16 anos (1981/ 1996) refletiu em mais gramas de bezerros produzido por quilo de vacas nos rebanhos $\mathrm{NeS}$ e $\mathrm{NeT}$, com diferenças em relação ao rebanho $\mathrm{NeC}$ de $4,7(\mathrm{NeS})$ e $6,8(\mathrm{NeT}) \mathrm{g} / \mathrm{kg}$ de vaca para R120 e 5,5 (NeS) e 11,7 $(\mathrm{NeT}) \mathrm{g} / \mathrm{kg}$ de vaca para R210, respectivamente.

Os coeficientes de regressão linear e seus níveis de significância por rebanho encontram-se na Tabela 3 Segundo DICKERSON (1969), esses coeficientes de regressão linear permitem obter estimativa da mudança genética anual, apresentando altos erros-padrão para o rebanho $\mathrm{NeC}$ nas características pesos dos bezerros e das vacas. Entretanto, expressam claramente tendência de resposta positiva, principalmente em $\mathrm{NeS}$ e $\mathrm{NeT}$, para essas características. Segue essa tendência a relação R210 para o rebanho NeT. Essas tendências ficam melhores ilustradas nas Figuras 1 a 6 , que apresentam as médias das características estudadas, em função de ano de nascimento dos bezerros.

A Figura 1 demonstra que houve tendência de

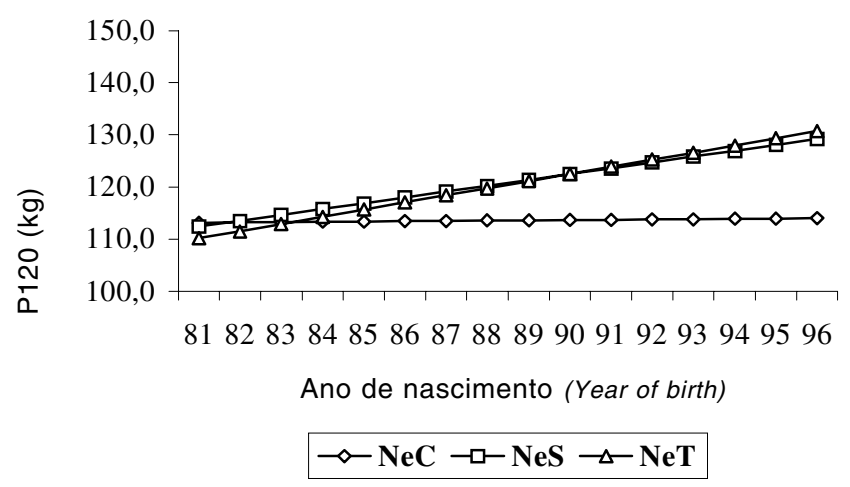

Figura 1 - Tendência fenotípica linear do P120 por ano de nascimento do bezerro nos rebanhos $\mathrm{NeC}, \mathrm{NeS}$ e NeT.

Figure 1 - Linear phenotypic trend of P120 on calf birth year for $\mathrm{NeC}, \mathrm{NeS}$ and $\mathrm{NeT}$ herds. 
1024 Rev. bras. zootec.

Tabela 3 - Coeficientes de regressão linear \pm erros-padrão $\left(b_{1} \pm E P\right)$ para características P120, P210, PV120, PV210, R120 e R210 por ano de nascimento das progênies nos rebanhos $\mathrm{NeC}$, NeS e $\mathrm{NeT}$

Table 3 - Linear regression coefficients \pm standard errors $\left(b_{1} \pm S E\right)$ of P120, P210, PV120, PV210, R120 and R210 on year of birth for NeC, $\mathrm{NeS}$ and $\mathrm{NeT}$ herd progenies

\begin{tabular}{llccccc}
\hline $\mathrm{Reb}^{2}$ & $\mathrm{P} 120(\mathrm{~kg})^{1}$ & $\mathrm{P} 210(\mathrm{~kg})^{1}$ & $\mathrm{PV} 120(\mathrm{~kg})^{1}$ & $\mathrm{PV} 210(\mathrm{~kg})^{1}$ & $\mathrm{R} 120(\mathrm{~g} / \mathrm{kg})^{1}$ & $\mathrm{R} 210(\mathrm{~g} / \mathrm{kg})^{1}$ \\
\cline { 2 - 7 } $\mathrm{Herd}^{2}$ & $\mathrm{~b}_{1} \pm \mathrm{EP}$ & $\mathrm{b}_{1} \pm \mathrm{EP}$ & $\mathrm{b}_{1} \pm \mathrm{EP}$ & $\mathrm{b}_{1} \pm \mathrm{EP}$ & $\mathrm{b}_{1} \pm \mathrm{EP}$ & $\mathrm{b}_{1} \pm \mathrm{EP}$ \\
& $b_{1} \pm S E$ & $b_{1} \pm S E$ & $b_{1} \pm S E$ & $b_{1} \pm S E$ & $b_{1} \pm S E$ & $b_{1} \pm S E$ \\
\hline $\mathrm{NeC}$ & $0,06 \pm 0,15$ & $0,33 \pm 0,23$ & $-1,09 \pm 0,44^{* *}$ & $-0,73 \pm 0,44$ & $0,89 \pm 0,41$ & $1,50 \pm 0,58^{* *}$ \\
$\mathrm{NeS}$ & $1,12 \pm 0,11^{* *}$ & $2,18 \pm 0,16^{* *}$ & $3,77 \pm 0,32^{* *}$ & $4,07 \pm 0,31^{* *}$ & $0,29 \pm 0,29$ & $1,36 \pm 0,41^{* *}$ \\
$\mathrm{NeT}$ & $1,37 \pm 0,09^{* *}$ & $2,31 \pm 0,14^{* *}$ & $2,00 \pm 0,27^{* *}$ & $1,65 \pm 0,27^{* *}$ & $1,77 \pm 0,25^{* *}$ & $3,61 \pm 0,35^{* *}$ \\
\hline
\end{tabular}

1 P120 e P210: pesos dos bezerros aos 120 e 210 dias de idade, respectivamente; PV120 e PV210: pesos das vacas nas respectivas idades dos bezerros; R120 e R210: P120/PV120 e P210/PV210, respectivamente.

$2 \mathrm{NeC}$ : rebanho controle não-selecionado; NeS e NeT: rebanhos selecionados para peso pós-desmama.

1 P120 e P210: calves weights at 120 and 210 days of age; PV120 and PV210: cows weights at correspondent ages of calves; R120 and R210: P120/PV120 and P210/PV210 respectively.

$2 \mathrm{NeC}$ : Unselected control population; NeS e NeT: Selected Nellore lines for postweaning weight.

maior aumento de P120 no decorrer dos anos, para os rebanhos $\mathrm{NeS}$ e $\mathrm{NeT}$ em relação ao $\mathrm{NeC}$. Os coeficientes de regressão estimados para os três rebanhos evidenciam tendências fenotípicas positivas para o $\mathrm{P} 120$, de 0,06, 1,12 e 1,37 kg/ano, respectivamente, para os rebanhos $\mathrm{NeC}$, NeS e $\mathrm{NeT}$. Para $\mathrm{NeC}$, considerada população controle, não-selecionada, o coeficiente de regressão linear foi bem inferior, o que evidencia ganho genético correlacionado para esse caráter no $\mathrm{NeS}$ e NeT.

Na Figura 2, observa-se também tendência crescente em P210, sendo que os coeficientes de regressão linear desse caráter, em função de ano de nascimento do bezerro, também foram bem superiores nos rebanhos $\mathrm{NeS}$ e $\mathrm{NeT}$, com valores respectivos de 2,18 e 2,31 kg/ano. O coeficiente de regressão linear para $\mathrm{NeC}$ foi de $0,33 \mathrm{~kg} / \mathrm{ano}$, evidenciando, portanto, ganho genético correlacionado nos rebanhos selecionados. $\mathrm{O}$ fato de o rebanho $\mathrm{NeT}$ apresentar-se superior, em alguns anos, em relação ao $\mathrm{NeS}$ possivelmente seja resultado, principalmente, do uso de touros provados pela progênie no rebanho NeS, para a característica peso aos 378 dias.

Para PV120 e PV210, mostrados nas Figura 3 e 4, notam-se grandes diferenças de pesos nos três rebanhos. O comportamento das médias de pesos das vacas ao desmame (PV210), visualizado na Figura 4, mostra que o rebanho $\mathrm{NeS}$ apresentou tendência de ganhos de peso superiores ao $\mathrm{NeC}$ e $\mathrm{NeT}$. Os coeficientes de regressão para os pesos das vacas apresentaram-se superiores para os rebanhos $\mathrm{NeS}$ e NeT, comparados ao $\mathrm{NeC}$, que, por sua vez, apresentou coeficientes de regressão linear negativos tanto para PV120 como para

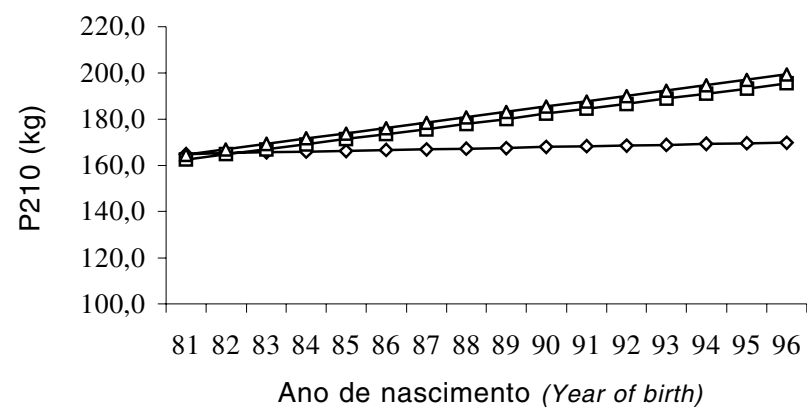

$\multimap-\mathrm{NeC} \rightarrow-\mathrm{NeS} \backsim-\mathrm{NeT}$

Figura 2 - Tendência fenotípica linear do P210 por ano de nascimento do bezerro nos rebanhos $\mathrm{NeC}, \mathrm{NeS}$ e NeT.

Figure 2 - Linear phenotypic trend of P210 on calf birth year for $\mathrm{NeC}, \mathrm{NeS}$ and $\mathrm{NeT}$ herds.

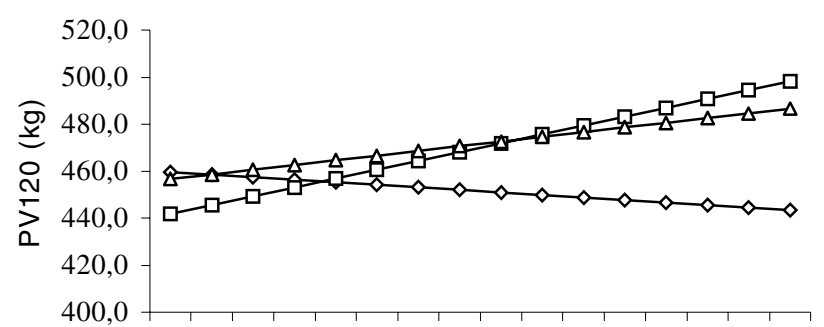

81828384858687888990919293949596

Ano de nascimento (Year of birth)

$$
\leadsto \mathrm{NeC} \rightarrow-\mathrm{NeS} \rightarrow-\mathrm{NeT}
$$

Figura 3 - Tendência fenotípica linear do PV120 por ano de nascimento do bezerro nos rebanhos $\mathrm{NeC}, \mathrm{NeS}$ e NeT.

Figure 3 - Linear phenotypic trend of PV120 on calf birth year for $\mathrm{NeC}, \mathrm{NeS}$ and $\mathrm{NeT}$ Nellore herds. 
SILVA et al.

PV210. As tendências fenotípicas positivas para NeS e NeT são decorrentes da seleção direta para maior peso aos 18 meses de idade, que foi o critério de seleção das fêmeas, refletindo-se em seus pesos adultos. Por outro lado, como a reposição no $\mathrm{NeC}$ foi feita pela média do peso aos 18 meses, verificou-se que os pesos das vacas, tanto aos 120 como aos 210 dias de idade do bezerro, tiveram tendência de estabilização, embora no início do processo. À medida que as vacas fundadoras do $\mathrm{NeC}$ eram substituídas, houve tendência negativa, ocasionada pela reposição de vacas pelo critério peso das vacas aos 550 dias de idade, o que é evidenciado na Tabela 4.

Com relação às tendências lineares das relações R120 e R210 (Figuras 5 e 6, respectivamente), verificou-se que em ambas idades houve tendência crescente nos três rebanhos, porém com maior evidência para o rebanho NeT. O componente linear apresentou coeficientes de determinação muito baixos com valor mais elevado para o rebanho NeT em R210. Isso evidencia que o comportamento das médias ajustadas para essas características apresentou-se extremamente variável, com tendência que nem sempre obedeceu à linearidade. As relações R120 e R210, por serem obtidas por razões que são alteradas constantemente, quer seja no denominador, representado pelo peso da vaca, que é alterado pela seleção direta, quer seja no numerador, representado pelo peso do bezerro, também alterado pela seleção indireta, tornam essa variável extremamente oscilante no decorrer dos anos, fazendo com que essas medidas sejam questionáveis para a avaliação da eficiência de vacas.

Objetivando visualizar a magnitude das mudanças nas médias dos pesos de bezerros e vacas e das relações às idades de 120 e 210 dias, foram calculadas médias para intervalos de períodos de nascimento dos bezerros, os quais foram referentes aos anos 1981/ 1985, 1988/1991 e 1992/1996 para os rebanhos NeC, $\mathrm{NeS}$ e NeT (Tabela 4). Essas médias, em relação ao rebanho $\mathrm{NeC}$, possibilitam comparar a tendência genética para essas características.

As tendências obtidas para os rebanhos $\mathrm{NeS}$ e $\mathrm{NeT}$, em relação ao $\mathrm{NeC}$, apresentaram-se todas positivas, com exceção do período de 1981/1985 no NeS, nos pesos de PV120 (- 1,8\%) e PV210 (- 0,7\%), e no NeT, para o PV120 (-1,6\%). Esta diferença, a favor do $\mathrm{NeC}$, provavelmente foi causada pela presença das vacas fundadoras neste rebanho. No entanto, após o ano de 1989, com descarte de todas as vacas fundadoras, as vacas do NeS tornaram-se superiores, refletindo o resultado da reposição, por

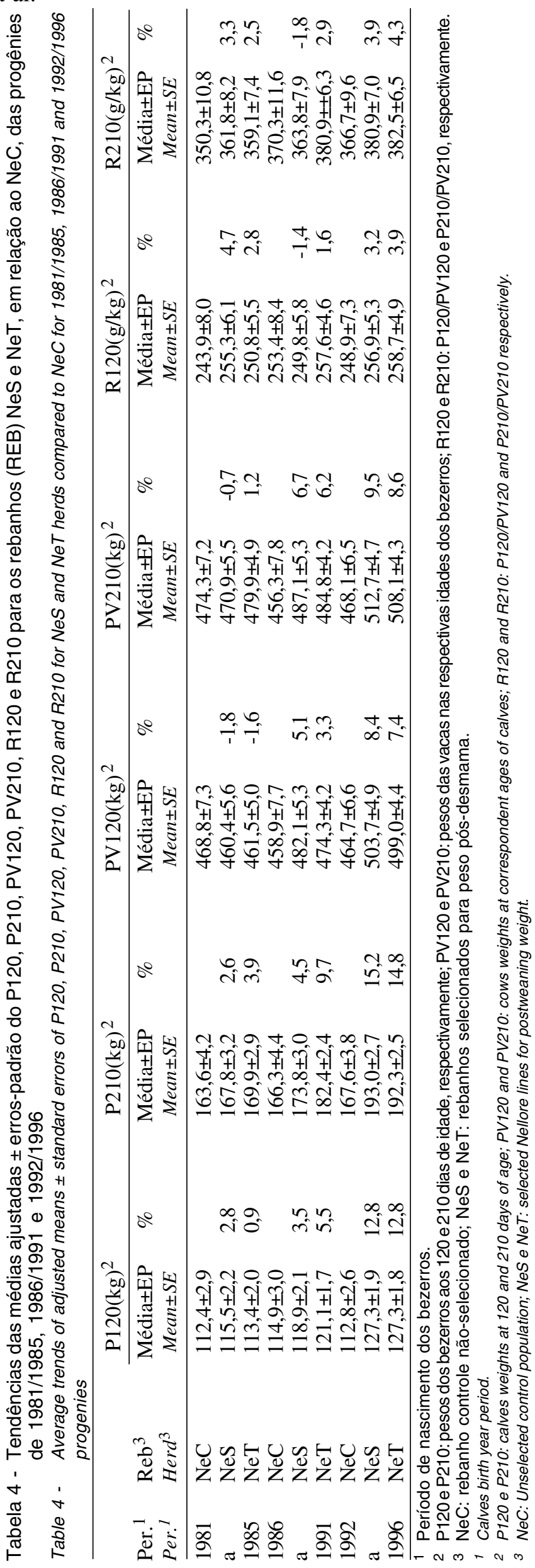


1026 Rev. bras. zootec.

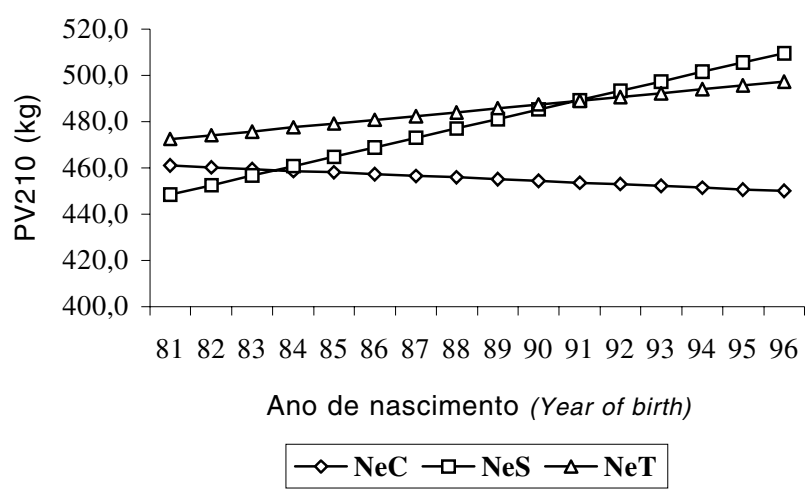

Figura 4 - Tendência fenotípica linear do PV210 por ano de nascimento do bezerro nos rebanhos $\mathrm{NeC}, \mathrm{NeS}$ e $\mathrm{NeT}$.

Figure 4 - Linear phenotypic trends of PV210 on calf birth year for $\mathrm{NeC}, \mathrm{NeS}$ and $\mathrm{NeT}$ Nellore herds.

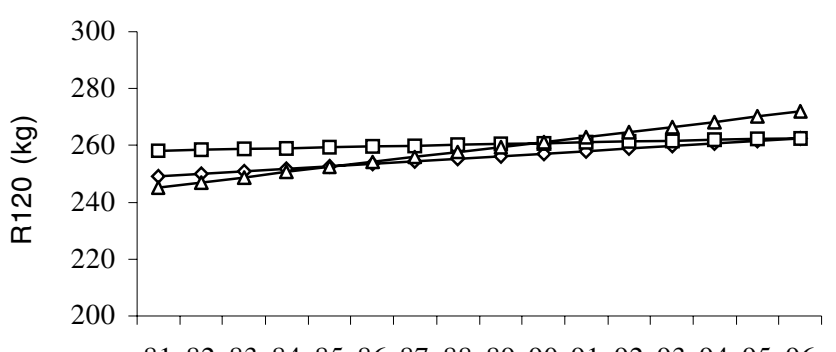

Ano de nascimento (Year of birth)

$\diamond-\mathrm{NeC} \rightarrow-\mathrm{NeS} \rightarrow-\mathrm{NeT}$

Figura 5 - Tendência fenotípica linear da relação R120 por ano de nascimento do bezerro nos rebanhos $\mathrm{NeC}, \mathrm{NeS}$ e $\mathrm{NeT}$.

Figure 5 - Linear phenotypic trends of $R 120$ ratio on calf birth year for $\mathrm{NeC}, \mathrm{NeS}$ and $\mathrm{NeT}$ herds.

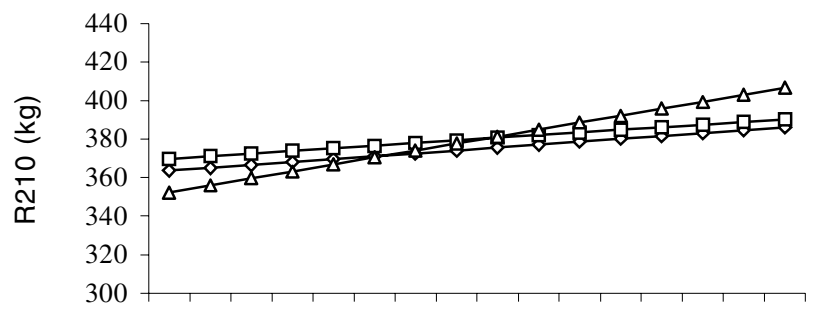

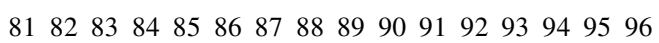

Ano de nascimento (Year of birth)

$$
\multimap \text { NeC } \square-\mathrm{NeS} \rightarrow \mathrm{NeT}
$$

Figura 6 - Tendência fenotípica linear da relação R210 por ano de nascimento do bezerro nos rebanhos $\mathrm{NeC}, \mathrm{NeS}$ e $\mathrm{NeT}$.

Figure 6 - Linear phenotypic trends of $R 210$ ratio on calf birth year for $\mathrm{NeC}, \mathrm{NeS}$ and $\mathrm{NeT}$ Nellore herds. meio de seleção no peso aos 18 meses de idade.

Os pesos das vacas nos últimos cinco anos, principalmente no rebanho NeS, o qual possui os maiores pesos, apresentaram superioridade de $8,4 \mathrm{e}$ 9,5\% para os pesos PV120 e PV210, respectivamente, em relação ao $\mathrm{NeC}$.

A Tabela 4 evidencia também acentuado acréscimo tanto no peso aos 120 como no peso aos 210 dias dos bezerros, o que ficou evidente principalmente no período 1992/96, que refletiu resposta indireta acumulada. O P210 aumentou em relação ao controle $(\mathrm{NeC}) 15,2$ e 14,8\%, respectivamente, para $\mathrm{NeS}$ e NeT.

As tendências de ganho, em termos percentuais em relação ao $\mathrm{NeC}$, das relações $\mathrm{R} 120$ e R210 foram positivas nos períodos 1981/1985 e 1992/1996 no rebanho $\mathrm{NeS}$ e nos três períodos para o NeT. A tendência negativa no período de $1986 / 1991$, para o rebanho $\mathrm{NeS}$, provavelmente deveu-se ao fato de o aumento no peso das vacas ter sido de maior magnitude, percentualmente, em relação ao aumento nos pesos dos bezerros.

A seleção para peso pós-desmama promoveu maiores P120 e P210, sugerindo aumento da habilidade materna, mas elevou também o peso das vacas, fazendo com que as R120 e R210 fossem alteradas em menor magnitude. Considerando-se os últimos cinco anos de produção de bezerros (1992/96), a superioridade dos rebanhos $\mathrm{NeS}$ e $\mathrm{NeT}$, em relação ao $\mathrm{NeC}$, foi, respectivamente, de 3,2 e $3,9 \%$ e 3,9 e 4,3\% para R120 e R210.

Assim, o processo de seleção no decorrer dos 16 anos refletiu em mais gramas de bezerro produzido por quilograma de vaca nos rebanhos NeS e NeT, tanto aos 120 como aos 210 dias de idade dos bezerros, em relação ao $\mathrm{NeC}$.

\section{Conclusões}

A seleção para peso pós-desmama na EEZS proporcionou ganho genético correlacionado nos pesos pré e à desmama dos bezerros.

A seleção com base no desempenho individual pós-desmama promoveu ganho nos pesos das vacas, porém, em termos percentuais, de menor magnitude que aquele observado para os pesos até a desmama dos bezerros.

A seleção para peso pós-desmama nos rebanhos da raça Nelore da EEZS produziu mais gramas de bezerro por quilograma de vaca, tanto aos 120 como aos 210 dias de idade de seus produtos. 


\section{Referências Bibliográficas}

ALENCAR, M.M. 1988. Desempenho produtivo de fêmeas das raças Canchim e Nelore. V. Desenvolvimento dos bezerros. R. Soc. Bras. Zootec., 17(4):411-420.

ALENCAR, M.M., BARBOSA, P.F., TREMATORE, R.L. et al. Peso ao parto, período de gestação e desempenho produtivo de vacas da raça Nelore e cruzadas Tabapuã x Gir. In: REUNIÃO ANUAL DA SOCIEDADE BRASILEIRA ZOOTECNIA, 33, 1996, Fortaleza. Anais... Fortaleza, 1996, p.130-132.

ALENCAR, M.M., TREMATORE, R.L.,BARBOSA, P.F. Composição ótima em composto de Charolês e Nelore baseada em estimativas de efeitos aditivos e heteróticos para pesos. In: REUNIÃO ANUAL DA SOCIEDADE BRASILEIRA ZOOTECNIA, 34, 1997, Juiz de Fora. Anais... Juiz de Fora, 1997, p.227-229.

ALVES FILHO, D.C., RESTLE, J. Efeito de grupo genético e da heterose na variação anual do peso e estado corporal de vacas com duas parições consecutivas. In: REUNIÃO ANUAL DA SOCIEDADE BRASILEIRA ZOOTECNIA, 33, 1996, Fortaleza. Anais... Fortaleza, 1996, p.193-195.

DAVIS, M.E., RUTLEDGE, J.J., CUNDIFF, L.V. et al. 1983. Life cycle efficiency of beef production: II. Relationship of cow efficiency ratios to traits of the dam and progeny weaned. J. Anim. Sci., 57(4):852-866.

DICKERSON, G.E. 1969. Techniques for research in quantitative animal genetics. In: Techniques and procedures in animal production research. New York. p.36-79.

DINKEL, C.A., BROWN, M.A. 1978. An evaluation of the ratio of calf weight to cow weight as an indicator of cow efficiency. J. Anim. Sci., 46(3):614-617.

DINKEL, C.A., MARSHALL, D.M., TUCKER, W.L. 1992. The breed of dam by sex of calf interaction effect on beef cattle weaning weight and cow efficiency. Can. J. Anim. Sci., 72:981-985.

EUCLIDES FILHO, K., FIGUEIREDO, G.R., SILVA, L.O.C. et al. Pesos ao nascer e à desmama e ganho pré desmama de Nelore e seus mestiços com Fleckvieh, Chianina, Charolês e Angus. In: REUNIÃ̃O ANUAL DA SOCIEDADE BRASILEIRA DE ZOOTECNIA, 33, Fortaleza, 1996. Anais... Fortaleza, p.164-166,1996.

FERRAZ FILHO, P.B., SOBRINHO, E.B., SILVA, L.O.C. et al. Tendência genética em pesos de bovinos da raça Nelore mocha no Brasil. In: REUNIÃO ANUAL DA SOCIEDADE BRASILEIRA ZOOTECNIA, 34, 1997, Juiz de Fora. Anais.. Juiz de Fora, p.254-256, 1997.

FRAHM, R.R., MARSHALL, D.M. 1985. Comparisons among two-breed cross cow groups. I. Cow productivity and calf performance to weaning. J. Anim. Sci., 61(4):844-855.

LÔBO, R.B., REYES, A. de LOS, BEZERRA, L.A.F. 1997. Avaliação genética de animais jovens, touros e matrizes. 1.ed. Ribeirão Preto: Departamento de Genética - FMRP - USP. 72p.

MACHADO, P.F.A., AQUINO, L.H., GONÇALVES, T.M. et al. Influência de fatores de meio sobre características produtivas de animais da raça Nelore. In: REUNIÃO ANUAL DA SOCIEDADE BRASILEIRA DE ZOOTECNIA, 34, Juiz de Fora, 1997. Anais... Juiz de Fora, p.211-213, 1997.

MAGNABOSCO, C.U., FAMULA, T.R., LÔBO, R.B. et al. Estimativas de parâmetros genéticos e de ambientes de características de crescimento em bovinos da raça Nelore. In: REUNIÃO ANUAL DA SOCIEDADE BRASILEIRA DE ZOOTECNIA, 33, Fortaleza, 1996. Anais... Fortaleza, p.142-144, 1996.

MARTINS FILHO, R., LOBO, R.N.B., LIMA, F.A.M. et al. Parâmetros genéticos e fenotípicos de pesos e ganhos em pesos de bovinos zebus no estado do Ceará. In: REUNIÃO ANUAL DA SOCIEDADE BRASILEIRA DE ZOOTECNIA, 34, Juiz de Fora, 1997. Anais... Juiz de Fora, p.248-250, 1997.

MICROSOFT EXCEL. 1994. Guia do usuário. 1.ed. Microsoft Corporation: Cambridge. 826p.

MORRIS, C.A., WILTON, J.W. 1976. Influence of body size on the biological efficiency of cows: A review. Can. J. Anim. Sci., 56:613-647.

OLIVEIRA, J.A.L., ALENCAR, M.M., LIMA, R. 1995. Eficiência produtiva de vacas da raça Nelore. R. Soc. Bras. Zootec., 24(3):445-452.

PEROTTO, D., CUBAS, A.C., ABRAHÃO, J.J.S. et al. Desempenho ponderal de animais Nelore e cruzas com Nelore. I. Período pré desmama. In: REUNIÃO ANUAL DA SOCIEDADE BRASILEIRA DE ZOOTECNIA, 33, Fortaleza, 1996. Anais... Fortaleza, p.124-126, 1996.

RAHNEFELD, G.W., WEISS, G.M., WARD, D. 1993. A comparison of methods to evaluate beef cow produtivity. Can. J. Anim. Sci., 73:971-975.

RAZOOK, A.G., FIGUEIREDO, L.A., NETO, L.M.B., et al. 1993. Intensidade de seleção e respostas direta e correlacionadas em 10 anos de progênies de bovinos das raças Nelore e Guzerá selecionadas para peso pós-desmame. Bol. Ind. Anim., 50(2):147-163.

SAS STATISTICAL ANALYSIS SYSTEMS INSTITUTE. 1992. Statistical analysis systems introductory guide for personal computers. 1.ed. Cary: NC. 111p.

SOUZA, J.C., EUCLIDES FILHO, K., SILVA, L.O.C. et al. Estimativas de parâmetros genéticos para o peso ao desmame de animais da raça Nelore no Brasil. In: REUNIÃO ANUAL DA SOCIEDADE BRASILEIRA ZOOTECNIA, 34, 1997, Juiz de Fora. Anais... Juiz de Fora, p.251-253, 1997.

URICK, J.J., KNAPP, B.W., BRINKS, J.S. et al. 1971. Relationships between cow weights and calf weaning weights in Angus, Charolais and Hereford breeds. J. Anim. Sci., 33(2):343-348.

Recebido em: 06/01/1999

Aceito em: 26/11/1999 